\title{
Serum adipokine levels in rheumatoid arthritis patients and their contributions to the resistance to treatment
}

\author{
KYOUNG SOO KIM ${ }^{1}$, HYUN-MI CHOI ${ }^{1}$, HYE-IN JI ${ }^{1}$, RAN SONG ${ }^{2}$, HYUNG-IN YANG ${ }^{2}$, \\ SOO-KON LEE ${ }^{3}$, MYUNG CHUL YOO ${ }^{1}$ and YONG-BEOM PARK ${ }^{3}$
}

\begin{abstract}
${ }^{1}$ East-West Bone and Joint Research Institute, Kyung Hee University Hospital at Gangdong, Kyung Hee University;
${ }^{2}$ Division of Rheumatology, Department of Internal Medicine, Kyung Hee University Hospital at Gangdong, Kyung Hee University Medical School, Gangdong-gu; ${ }^{3}$ Division of Rheumatology, Department of Internal Medicine, Yonsei University College of Medicine, Seodaemoon-gu, Seoul, Republic of Korea
\end{abstract}

Received April 28, 2012; Accepted October 17, 2013

DOI: $10.3892 / \mathrm{mmr} .2013 .1764$

\begin{abstract}
The aim of this study was to determine whether disease activity and the type of therapy differentially modulate serum adipokine levels in patients with rheumatoid arthritis (RA), and whether pre-therapy adipokine levels contribute to resistance to treatment. Fasting blood samples from 40 RA patients were obtained at baseline and six months following therapeutic treatment with disease-modifying antirheumatic drugs (DMARDs) and/or tumor necrosis factor (TNF)- $\alpha$ blockers. Serum levels of adiponectin, leptin, visfatin and resistin were measured by ELISA. Baseline adipokine levels did not exhibit a statistically significant difference when comparing patients with moderate and high disease activity, based on the disease activity score in 28 joints (DAS28). Of all the adipokines, only adiponectin was significantly increased in patients responding to DMARDs and/or TNF- $\alpha$ blocker therapy, based on the American College of Rheumatology $20 \%$ improvement criteria (ACR20) at six months $(2,964 \pm 1,237$ to $3,683 \pm 1,511 \mathrm{ng} / \mathrm{ml}, \mathrm{P}<0.01)$. However, adiponectin levels in non-responders did not significantly increase $(3,192 \pm 2,090$ to $3,222 \pm 1,150 \mathrm{ng} / \mathrm{ml}$ ). By contrast, there were no statistically significant changes in leptin, resistin or visfatin levels in either the responders or non-responders. Serum adipokine (adiponectin, leptin, visfatin, and resistin) levels in RA patients did
\end{abstract}

Correspondence to: Professor Kyoung Soo Kim, East-West Bone and Joint Research Institute, Kyung Hee University Hospital at Gangdong, Kyung Hee University, 149 Sangil-dong, Gangdong-gu, Seoul 135-747, Republic of Korea

E-mail: labrea46@naver.com

Dr Yong-Beom Park, Divsion of Rheumatology, Department of Internal Medicine, Yonsei University College of Medicine, 250 Sungsanno, Seodaemoon-gu, Seoul 120-752, Republic of Korea

E-mail: yongbpark@yuhs.ac

Key words: adipokine, adiponectin, disease-modifying antirheumatic drugs, tumor necrosis factor- $\alpha$ blocker, rheumatoid arthritis not significantly change following therapy, with the exception of adiponectin. Adipokine levels may not contribute to therapeutic resistance to DMARDs and/or TNF- $\alpha$ blocking agents.

\section{Introduction}

White adipose tissue (WAT) was, until recently, only regarded as a predominant site of energy storage with important roles in the control of energy homeostasis. However, WAT is now considered to be a predominant endocrine organ in humans, secreting a wide range of biologically active molecules, including numerous adipokines (1). A number of these adipokines, which are predominantly produced by WAT, exhibit the ability to modulate metabolic and inflammatory processes and are hypothesized to contribute to the pathophysiology of obesity-linked diseases (2). In particular, articular adipose tissue (AAT) is highly reactive, secreting considerable quantities of pro-inflammatory and anti-inflammatory cytokines and classical adipokines when stimulated (3). Thus, all the predominant adipose-derived products, termed adipokines, participate in inflammation and immunity. These include leptin, adiponectin, visfatin and resistin.

Adipokine levels are elevated in the sera and synovial fluids of patients with rheumatoid arthritis (RA). This suggests that adipokines are involved in the pathogenesis of RA by exerting potent modulatory effects on target tissues and cells involved in rheumatic disease, including cartilage, synovium, bone, and various immune cells $(4,5)$. Adiponectin also induces the in vitro production of pro-inflammatory cytokines, including interleukin (IL)-6, matrix metalloproteinase (MMP)-1 and IL-8 from RA synovial fibroblasts $(6,7)$. Thus, adiponectin is hypothesized to exert significant pro-inflammatory and matrix-degrading effects. Leptin stimulates T cell-mediated immunity, cytokine release from monocytes/macrophages and the differentiation of hematopoietic cells (8). Visfatin is a novel mediator of innate immunity. It is key in the persistence of inflammation through its capacity to inhibit neutrophil apoptosis (9). Furthermore, visfatin activates transcription factors, including nuclear factor (NF)- $\kappa \mathrm{B}$ and activator protein (AP)-1 and induces the production of IL-6, IL-8, MMP-1 and MMP-3 in RA synovial fibroblasts and IL-6 and tumor necrosis factor (TNF) in monocytes (10). 
Table I. Clinical and demographic characteristics of the study population.

\begin{tabular}{lccc}
\hline Characteristics & All RA patients & Responders & Non-responders \\
\hline Patients, $\mathrm{n}(\%)$ & 40 & $30(75)$ & $10(25)$ \\
Mean age \pm SD (years) & $45.2 \pm 13.6$ & $45.4 \pm 14.2$ & $44.5 \pm 12.2$ \\
Gender (female/male) & $32 / 8$ & $24 / 6$ & $8 / 2$ \\
Disease duration, months (range) & $5.2(1-12)$ & $5.5(1-12)$ & $4.6(2-8)$ \\
RF-positive, $\mathrm{n}(\%)$ & $38(95)$ & $28(93)$ & $10(100)$ \\
Anti-CCP positive, $\mathrm{n}(\%)$ & $36(90)$ & $27(90)$ & $9(90)$ \\
Baseline DAS28 score & $5.38 \pm 1.0$ & $5.65 \pm 0.9^{\mathrm{a}}$ & $4.54 \pm 0.7$ \\
HAQ-DI score & $0.96 \pm 0.5$ & $1.03 \pm 0.5$ & $0.75 \pm 0.6$ \\
Anti-TNF- $\alpha$ blocker use, $\mathrm{n}(\%)$ & $16(40)$ & $15(50)$ & $1(10)$ \\
\hline
\end{tabular}

${ }^{\text {a }} \mathrm{P}<0.05$, compared with non-responders. RA, rheumatoid arthritis; n, number of subjects; SD, standard deviation; RF, rheumatoid factor; anti-CCP, anti-cyclic citrullinated peptide antibodies; DAS28, disease activity score in 28 joints; HAQ-DI, Health Assessment Questionnaire disability index; TNF- $\alpha$, tumor necrosis factor- $\alpha$.

Human resistin also has pro-inflammatory functions, including the activation of NF- $\mathrm{NB}$-dependent pathways to produce TNF- $\alpha$, IL- 6 and IL- $1 \beta$ in human peripheral blood mononuclear cells (11). It is widely accepted, that adipokines are important in the pathogenesis of RA, yet the in vivo role of adipokines and their association with disease activity is poorly understood. Additional insight into the role of adipokines may aid in the development of novel therapeutic agents. In this study, the modulation of serum adipokines (adiponectin, leptin, resistin and visfatin) was evaluated depending on RA disease activity or the type of therapy, including disease-modifying antirheumatic drugs (DMARDs) and TNF- $\alpha$ blockers.

\section{Patients and methods}

Patients. A total of 40 RA patients (32 females and 8 males; mean age \pm SD, 45.2 \pm 13 .6 years), who fulfilled the 1987 revised criteria of the American College of Rheumatology (ACR) for RA (12), were evaluated prior to and six months following RA therapy (Table I). All the patients were consecutively admitted to the outpatient clinic of the Rheumatology Division of the Yonsei University Hospital (Seoul, Korea) and were treated with DMARDs: Monotherapy or combination therapy with methotrexate, sulfasalazine and hydroxychloroquine. In addition, 16 patients were also treated with TNF- $\alpha$ blockers [such as eternacept $\left(\right.$ Enbrel $^{\circledR}$; Pfizer, New York, NY, USA), infliximab (Remicade ${ }^{\circledR}$; Janssen Biotech, Horsham, PA, USA), adalimumab (Humira ${ }^{\circledR}$; Abbott Laboratories, Chicago, IL, USA)]. Rheumatoid factor (RF) and anti-cyclic citrullinated peptide antibodies (anti-CCP) were measured at the baseline. Disease activity was assessed according to the disease activity score in 28 joints (DAS28) based on C-reactive protein level (CRP) at baseline and six months following treatment (13). Health-related quality of life was evaluated using the patient-reported Health Assessment Questionnaire disability index (HAQ DI) (14). The therapeutic response was evaluated six months following treatment, according to the ACR response criteria (15). Patients were categorized as responders or non-responders, on the basis of fulfilling the ACR $20 \%$ improvement criteria (achieving an ACR20 response) six months following treatment. Serum samples were collected prior to (baseline) and six months following therapy. The present study was conducted in accordance with the recommendations of the Declaration of Helsinki and was approved by the Institutional Review Board of the Yonsei University Hospital. All the patients provided written informed consent.

Measurement of serum adipokine levels by ELISA. For the assessment of adiponectin, leptin, resistin and visfatin levels in serum, the serum was diluted with diluent buffer (BD OptEIA; BD Bioscience, San Diego, CA, US) for the proper detection range with ELISA, according to the manufacture's protocol. The adipokine levels in serum were measured using a commercial ELISA kit. Adiponectin and leptin ELISA kits were purchased from R\&D Systems (Minneapolis, MN, USA). Resistin and visfatin ELISA kits were purchased from Millipore (Billerica, MA, USA) and BioVision (Mountain View, CA, USA), respectively.

Statistical analysis. Serum adipokine levels at baseline DAS28 were compared using the Mann-Whitney U test (two-tailed). Pre- and post-treatment levels were compared using a Wilcoxon signed rank test (two-tailed). Data are expressed as the mean $\pm \mathrm{SD}$. The differences between the groups were compared using the Mann-Whitney U test (two-tailed). Prism software 4 (Graphpad Software, San Diego, CA, USA) was used for statistical analysis and graphing. $\mathrm{P}<0.05$ was considered to indicate a statistically significant difference.

\section{Results}

Baseline demographics and clinical characteristics. As shown in Table I, the majority of patients were female and had active disease (DAS28 mean \pm SD, 5.38 \pm 1.0 ) prior to therapy. Patients were recently diagnosed with $\mathrm{RA}$, having a mean disease duration of 5.2 months. Following DMARD and/or TNF- $\alpha$ blocker therapy, 30 (75\%) patients were responders and 10 (25\%) were non-responders. Of the responders, 15 were treated with DMARDs only, whereas another 15 were treated with DMARDs followed by a TNF- $\alpha$ blocker. Baseline demographics 
A

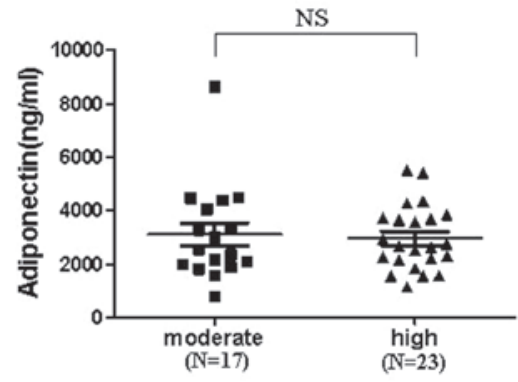

B

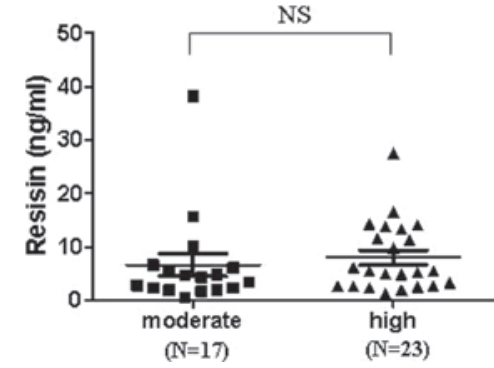

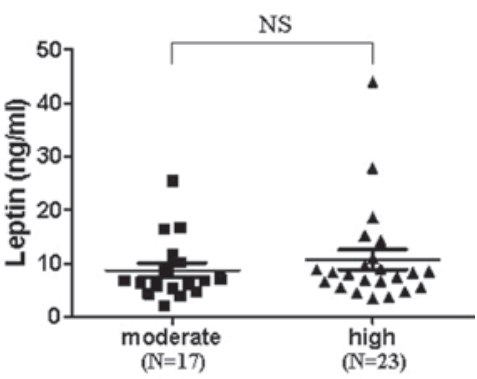

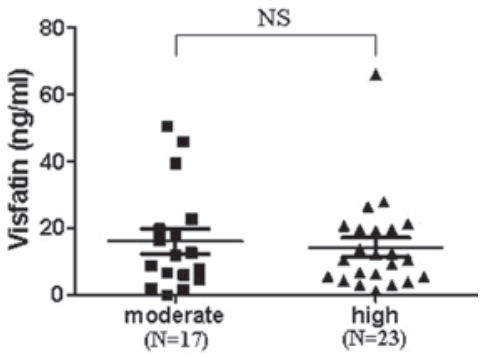

Figure 1. Comparison of serum adipokine levels of patients with moderate and high rheumatoid arthritis (RA) disease activity. In total, 40 patients diagnosed with RA prior to treatment (DAS28 baseline) were divided into moderate $(\mathrm{n}=17)$ or high disease activity $(\mathrm{n}=25)$ groups. Serum was analyzed for levels of adiponectin, leptin, resistin and visfatin. Serum samples were collected prior to therapy (baseline). RA, rheumatoid arthritis; DAS28, disease activity score in 28 joints; NS, not significant.

A

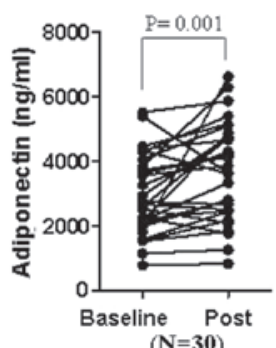

$(\mathbf{N}=\mathbf{3 0})$

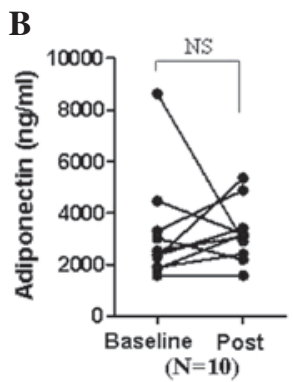

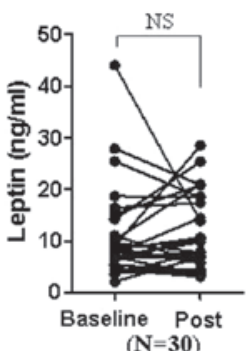

(N=30)

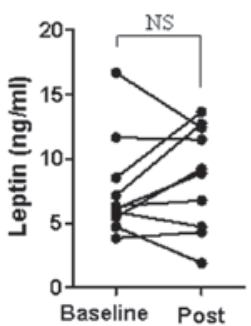

(N=10)

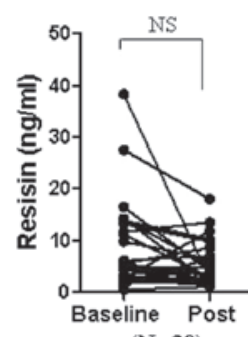

( $\mathbf{N}=\mathbf{3 0}$ )

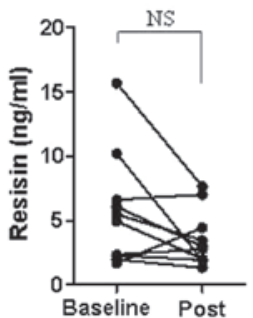

(N=10)

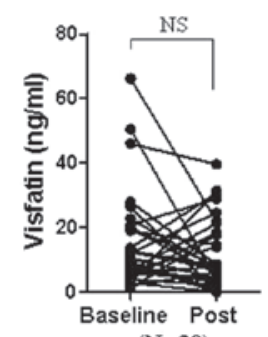

$(\mathrm{N}=30)$

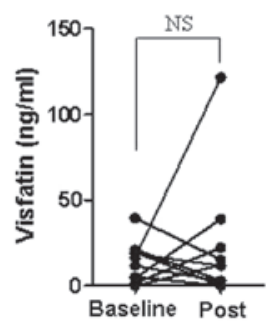

$(\mathrm{N}=10)$

Figure 2. Change in serum adipokine levels in (A) responders $(n=30)$ and $(B)$ non-responders $(n=10)$ prior to and following treatment. Responders showed the therapeutic effect of DMARDs and/or TNF- $\alpha$ blockers. The non-responder group did not show any therapeutic effect following DMARD and/or TNF- $\alpha$ blocker treatment ACR20 six months following treatment. Serum samples were collected prior to (baseline) and following six months of therapy (post). DMARDs, disease-modifying antirheumatic drugs; TNF- $\alpha$, tumor necrosis factor- $\alpha$; ACR20, American College of Rheumatology $20 \%$ improvement criteria; NS, not significant.

and clinical characteristics were similar between responders and non-responders, with the exception of the baseline DAS28 score, which was significantly higher in the 30 responders than in the 10 non-responders $(5.65 \pm 0.9$ vs. $4.54 \pm 0.7 ; \mathrm{P}<0.05)$. Due to a relatively higher disease activity, a greater number of patients in the responder group received TNF- $\alpha$ blocker therapy compared with the non-responder group.
Effect of disease activity on serum adipokine levels. To determine how adipokine levels are regulated in patients with RA depending on disease activity, serum was collected prior to (at baseline) and six months following therapy. Adipokine levels in the sera of patients with moderate disease activity on the basis of DAS28 were not significantly different from that of patients with high disease activity (Fig. 1). Thus, the 


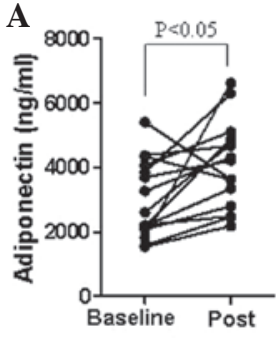

$(\mathrm{N}=\mathbf{1 5})$

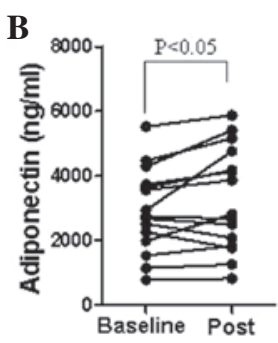

$(\mathrm{N}=\mathbf{1 5})$

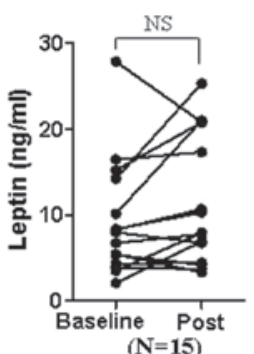

$(\mathrm{N}=15)$

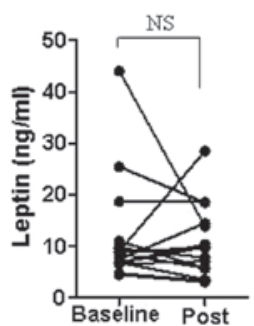

$(\mathrm{N}=\mathbf{1 5})$

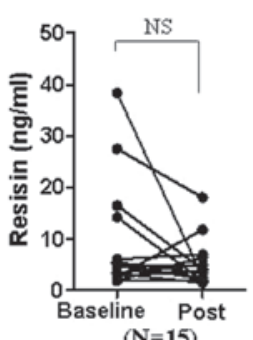

$(\mathbf{N}=\mathbf{1 5})$

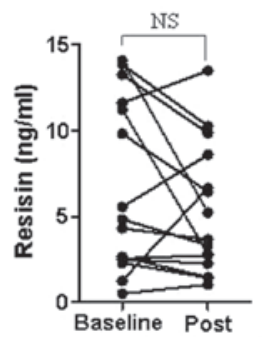

$(\mathrm{N}=\mathbf{1 5})$

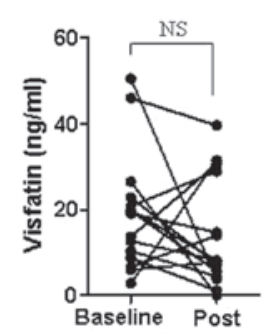

$(\mathbf{N}=\mathbf{1 5})$

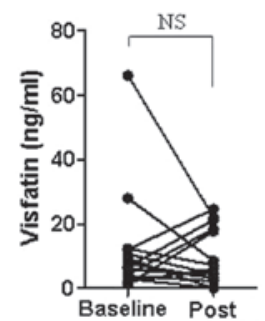

$(\mathrm{N}=\mathbf{1 5})$

Figure 3. Comparison of serum adipokine levels in responders receiving (A) disease-modifying antirheumatic drug (DMARD) treatment only (n=15) and (B) DMARD treatment followed by tumor necrosis factor- $\alpha(\mathrm{TNF}-\alpha)$ blocker therapy $(\mathrm{n}=15)$. Serum samples were collected prior to (baseline) and following six months of therapy (post). DMARDs, disease-modifying antirheumatic drugs; TNF- $\alpha$, tumor necrosis factor- $\alpha$; NS, not significant.

change in serum adipokine levels prior to and following the therapeutic treatment of patients, who responded well to DMARD and/or TNF- $\alpha$ blocker treatment in terms of ACR20 was examined (Fig. 2). Adiponectin levels were significantly elevated in responders subsequent to treatment (mean $\pm \mathrm{SD}$; from $2,964 \pm 1,237$ to $3,683 \pm 1,511 \mathrm{ng} / \mathrm{ml}, \mathrm{P}=0.001$ ), while adiponectin levels in non-responders revealed no statistically significant difference $(3,192 \pm 2,090$ to $3,222 \pm 1,150)$. By contrast, leptin, resistin and visfatin levels were not significantly changed in either responders or non-responders.

Effect of the type of therapeutic agent on serum adipokine levels. To determine whether the type of therapeutic agent differentially affects adipokine levels, the responder group was subdivided into patients receiving only DMARDs and patients treated with DMARDs followed by TNF- $\alpha$ blockers (Fig. 3). Adiponectin levels in the DMARD only group $(3,008 \pm 1,209$ to $4,089 \pm 1,345 \mathrm{ng} / \mathrm{ml})$ showed a greater increase compared with those of the DMARD+TNF- $\alpha$ blocker group $(2,921 \pm 1,305$ to $3,278 \pm 1,603 \mathrm{ng} / \mathrm{ml})$ following treatment, while other adipokine levels were not significantly affected.

Effect of pre-treatment adipokine levels on resistance to treatment. The effect of pre-treatment adipokine levels on resistance to treatment was also investigated. No statistically significant difference was identified in adipokine levels at baseline between responders and non-responders, suggesting that the responsiveness to therapeutic treatments was not affected by adipokine levels (data not shown).

\section{Discussion}

In this study, the effects of RA disease activity and type of therapeutic agent on serum adipokine levels was evaluated, as well as the correlation between pre-treatment adipokine levels and resistance to treatment. It was observed that only the level of adiponectin was significantly increased following therapeutic treatment, while other adipokine levels were not identified to be significantly different. Increased adiponectin was observed in patients treated with DMARDs alone or with a combination of DMARDs and TNF- $\alpha$ blockers. However, the level of adipokines at baseline was not significantly different between patients with moderate versus high disease activity. This suggests that moderate versus high RA disease activity does not significantly affect adipokine expression, as the two patient groups exhibited significant in vivo inflammation at baseline.

Previous studies have demonstrated that adipokine levels are affected by disease activity. DMARD treatment increased serum adiponectin levels in RA patients (16). TNF- $\alpha$ blocker therapy also increased adiponectin levels in a female RA patient three months following treatment (17). Other studies have shown that anti-TNF- $\alpha$ therapy significantly increases serum adiponectin levels two and six weeks following therapy in RA patients (18). Contradictory to these results, another study reported that a short- or longterm TNF- $\alpha$ blockade alone had no affect on circulating leptin and adiponectin concentrations in RA patients, while patients treated with anti-TNF- $\alpha$ and concomitant corticosteroids on a regular basis demonstrated a significant decrease in adiponectin levels six months after therapy (19). Furthermore, visfatin was not associated with inflammation in patients with severe RA undergoing anti-TNF- $\alpha$ therapy (20). Another study reported that anti-TNF- $\alpha$ therapy resulted in a rapid reduction in serum resistin levels, but did not modulate leptin levels in RA patients (21). It was also demonstrated that serum leptin concentrations are inversely correlated with inflammation in RA (22). The increased adipokine levels post-treatment in these studies 
is concurrent with the results of the present study; however, in order to clarify data concerning additional adipokines, further studies are required. Thus, it is currently difficult to delineate the behavior of these adipokines during the course of the disease or the way in which they are affected by treatment. In line with this, the resistin level in the serum and joint fluid of patients with RA was significantly higher compared with that in osteoarthritis (OA) patients (23). Serum adiponectin levels in RA patients were higher than those observed in the healthy controls, although not significantly different from those of OA patients (24). Serum adiponectin levels in RA patients were significantly lower compared with the synovial fluid levels, regardless of increased local inflammation in the joint. A previous study has demonstrated that serum adiponectin levels in RA patients are higher compared with OA patients, which is contradictory to the findings mentioned previously, stating that decreased disease activity or inflammation increases serum adiponectin levels. In addition, leptin levels in the joint fluid of patients with OA were significantly higher than the serum levels (23).

Certain criticisms may be raised regarding the parameters used for disease activity. In this study, the correlation analysis focused on DAS28 and additional parameters were not compared systematically. DAS28 may not be an ideal parameter to analyze disease activity over time. It is usually accepted that DAS28 is not an ideal parameter as it only focus on 28 joints excluding foot joints. Also, it focus on disease activity but not radiographic damage. Thus, doctors still require more developed parameters to evaluate disease degree. Thus, DAS28 in combination with analysis of radiographic damage and other parameters may be a useful application of adipokine levels with regard to disease progression. Furthermore, additional parameters to consider include: Age, body mass index (BMI) (with/without normalization), disease duration, CRP, association with RF/anti-CCP, proinflammatory cytokines, radiographic damage and gender. Leptin levels demonstrate statistically significant gender-specific differences (23), while adiponectin is unrelated to age, disease duration, BMI or disease activity in RA patients (24). In addition, systemic values of metabolically relevant factors, such as adipokines, may not reflect the situation in a specific organ or tissue under pathophysiological conditions, including the joint.

Adiponectin with low molecular weight has anti-inflammatory effects, whereas its globular form with high molecular weight exhibits pro-inflammatory effects (25). The various adiponectin molecular species are differentially generated depending on in vivo physiological conditions, although no previous study has assessed the ratios between these three forms in patients with RA. These results indirectly indicate that inflammation may not be crucial for the modulation of the in vivo adipokine expression pattern of RA patients, and various unknown factors may be more involved in the modulation of in vivo adipokine expression. In order to evaluate the correlation between adipokine levels and resistance to treatment, pre-therapeutic serum adipokine levels were analyzed. As there was no statistically significant difference between non-responders and responders, it was concluded that pre-therapeutic adipokine levels do not contribute to resistance to treatment.
In conclusion, serum adipokine levels in RA patients were not significantly altered following therapy, with the exception of adiponectin. The results suggest that serum adipokine levels do not affect the degree of resistance to treatment and that serum adipokine levels in RA patients may be affected by various cell types and other unknown factors. Thus, it was hypothesized that in RA, serum adipokine levels do not reflect and have a limited effect on intraarticular inflammation.

\section{Acknowledgements}

This study was supported by the Basic Science Research Program through the National Research Foundation of Korea (NRF) funded by the Ministry of Education, Science, and Technology (grant nos. 2011-0026939 and 2011-0009061) and Korea Healthcare Technology R\&D Project, Ministry of Health and Welfare, Republic of Korea (grant no. A102065).

\section{References}

1. Trayhurn P and Wood IS: Adipokines: inflammation and the pleiotropic role of white adipose tissue. Br J Nutr 92: 347-355, 2004.

2. Wozniak SE, Gee LL, Wachtel MS and Frezza EE: Adipose tissue: the new endocrine organ? A review article. Dig Dis Sci 54: 1847-1856, 2009

3. Kontny E, Plebanczyk M, Lisowska B, et al: Comparison of rheumatoid articular adipose and synovial tissue reactivity to proinflammatory stimuli: contribution to adipocytokine network. Ann Rheum Dis 71: 262-267, 2012.

4. Gomez R, Conde J, Scotece M, et al: What's new in our understanding of the role of adipokines in rheumatic diseases? Nat Rev Rheumatol 7: 528-536, 2011.

5. Neumann E, Frommer KW, Vasile M and Müller-Ladner U: Adipocytokines as driving forces in rheumatoid arthritis and related inflammatory diseases? Arthritis Rheum 63: 1159-1169, 2011.

6. Ehling A, Schaffler A, Herfarth $\mathrm{H}$, et al: The potential of adiponectin in driving arthritis. J Immunol 176: 4468-4478, 2006.

7. Kitahara K, Kusunoki N, Kakiuchi T, Suguro T and Kawai S: Adiponectin stimulates IL-8 production by rheumatoid synovial fibroblasts. Biochem Biophys Res Commun 378: 218-223, 2009.

8. Stofkova A: Leptin and adiponectin: from energy and metabolic dysbalance to inflammation and autoimmunity. Endocr Regul 43: $157-168,2009$.

9. Luk T, Malam Z and Marshall JC: Pre-B cell colony-enhancing factor (PBEF)/visfatin: a novel mediator of innate immunity. J Leukoc Biol 83: 804-816, 2008.

10. Brentano F, Schorr O, Ospelt C, et al: Pre-B cell colony-enhancing factor/visfatin, a new marker of inflammation in rheumatoid arthritis with proinflammatory and matrix-degrading activities. Arthritis Rheum 56: 2829-2839, 2007.

11. Bokarewa M, Nagaev I, Dahlberg L, Smith U and Tarkowski A: Resistin, an adipokine with potent proinflammatory properties. J Immunol 174: 5789-5795, 2005.

12. Arnett FC, Edworthy SM, Bloch DA, et al: The American Rheumatism Association 1987 revised criteria for the classification of rheumatoid arthritis. Arthritis Rheum 31: 315-324, 1988.

13. Inoue E, Yamanaka $\mathrm{H}, \mathrm{Hara} \mathrm{M}$, Tomatsu $\mathrm{T}$ and Kamatani $\mathrm{N}$ : Comparison of Disease Activity Score (DAS)28- erythrocyte sedimentation rate and DAS28- C-reactive protein threshold values. Ann Rheum Dis 66: 407-409, 2007.

14. Fries JF, Spitz P, Kraines RG and Holman HR: Measurement of patient outcome in arthritis. Arthritis Rheum 23: 137-145, 1980.

15. Felson DT, Anderson JJ, Boers M, et al: American College of Rheumatology. Preliminary definition of improvement in rheumatoid arthritis. Arthritis Rheum 38: 727-735, 1995.

16. Cansu B, Cansu DU, Kaşifoglu T, Gülbas $Z$ and Korkmaz C: Disease-modifying antîrheumatic drugs increase serum adiponectin levels in patients with rheumatoid arthritis. J Clin Rheumatol 17: 14-17, 2011. 
17. Lewicki M, Kotyla P and Kucharz E: Etanercept increases adiponectin level in woman with rheumatoid arthritis. Clin Rheumatol 27: 1337-1338, 2008.

18. Komai N, Morita Y, Sakuta T, Kuwabara A and Kashihara $\mathrm{N}$ : Anti-tumor necrosis factor therapy increases serum adiponectin levels with the improvement of endothelial dysfunction in patients with rheumatoid arthritis. Mod Rheumatol 17: 385-390, 2007

19. Popa C, Netea MG, de Graaf J, et al: Circulating leptin and adiponectin concentrations during tumor necrosis factor blockade in patients with active rheumatoid arthritis. J Rheumatol 36: 724-730, 2009

20. Gonzalez-Gay MA，Vazquez-Rodriguez TR, Garcia-Unzueta MT, et al: Visfatin is not associated with inflammation or metabolic syndrome in patients with severe rheumatoid arthritis undergoing anti-TNF-alpha therapy. Clin Exp Rheumatol 28: 56-62, 2010.
21. Gonzalez-Gay MA, Garcia-Unzueta MT,Gonzalez-Juanatey C, et al: Anti-TNF-alpha therapy modulates resistin in patients with rheumatoid arthritis. Clin Exp Rheumatol 26: 311-316, 2008.

22. Popa C, Netea MG, Radstake TR, et al: Markers of inflammation are negatively correlated with serum leptin in rheumatoid arthritis. Ann Rheum Dis 64: 1195-1198, 2005.

23. Presle N, Pottie P, Dumond H, et al: Differential distribution of adipokines between serum and synovial fluid in patients with osteoarthritis. Contribution of joint tissues to their articular production. Osteoarthritis Cartilage 14: 690-695, 2006.

24. Senolt L, Pavelka K, Housa D and Haluzik M: Increased adiponectin is negatively linked to the local inflammatory process in patients with rheumatoid arthritis. Cytokine 35: 247-252, 2006

25. Neumeier M, Weigert J, Schäffler A, et al: Different effects of adiponectin isoforms in human monocytic cells. J Leukoc Biol 79: 803-808, 2006. 